\title{
A Watermark Evaluation Bench for Content Adaptation Modes
}

\author{
Deepayan Bhowmik and Charith Abhayaratne
}

Dept. of Electronic and Electrical Engineering, University of Sheffield, UK. \{d.bhowmik, c.abhayaratne\}@ sheffield.ac.uk.

Keywords: watermarking, wavelets, content adaptation.

\begin{abstract}
Watermarks embedded in scalable coded media have to be robust to content adaptation (CA) to address network, display and usage requirements. A modularised flexible formal framework as a watermark evaluation bed for content adaptation modes (WEBCAM) is presented. WEBCAM-phase 1 allows evaluation of the effect of various design parameters in wavelet-based watermarking on robustness to CA.
\end{abstract}

\section{Introduction}

Seamless delivery of media content from production to users is vital in multimedia applications. After content production, they have to be transmitted to the user via communication networks with various bandwidth capabilities to display devices with various display resolutions and available resources addressing various usage requirements. This requires the content to be adapted according to these requirements along the media supply chains. This is usually achieved by scalable encoding for content at the time of media production and then adapting the content by truncating the scalable bit stream at the various points of the delivery chain. Current scalable coders for visual content include JPEG2000 and the emerging H.264 scalable video coding (SVC) extension. They provide the quality and the spatio-temporal resolution scalability for content adaptation. In the course of content adaptation, any watermarking data, which is embedded in the visual content at the time of media production, for digital rights management (DRM) proposes are also altered and consequently it affects the content authentication in DRM. Therefore, it is vital to make watermarking robust to content adaptation attacks.

Recent years have seen a plethora of visual media watermarking algorithms being developed with the advancement of visual media technologies. However, there is only little effort has been made on formal evaluation of such watermarking techniques [1], There is a necessity for the evaluation of the watermark robustness to content adaptations as the use of scalable coding at media production is becoming popular. In this paper, we present the development of a watermark evaluation bed for content adaptation modes (WEBCAM), a flexible modular formal framework for evaluating the effect of various design parameters involved in wavelet-based watermarking and the associated detection and authentication procedures on the watermark robustness to content adaptation attacks.

\section{WEBCAM Systems Architecture}

The WEBCAM system consists of three parts, namely, 1. Watermark embedding, 2. Content adaptation, and 3. Watermark extraction and authentication as shown in Figure 1.

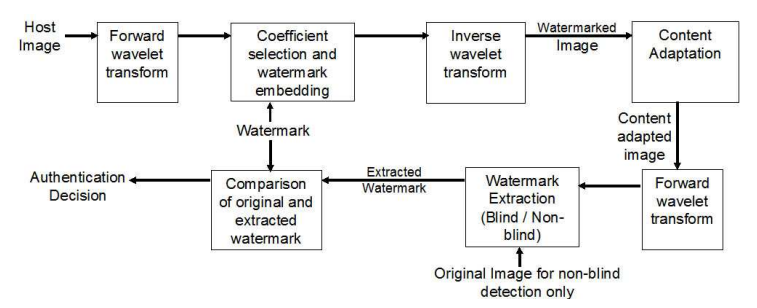

Figure 1: WEBCAM systems architecture block diagram.

Watermark Embedding (WE) focuses on wavelet-based watermarking by dissecting algorithms into the choice of wavelet kernel (e.g., orthogonal, biorthogonal, Morphological and spatially adaptive wavelets), the choice wavelet sub band and coefficient selection for watermark embedding and the embedding method (e.g., quantisation, direct coefficient modification, etc.). This allows us to pick and mix choices for each of the design parameters and generate new sets of watermarking algorithms. WE also computes the embedding performance metrics such as imperceptibility measure and data hiding capacity.

Content Adaptation (CA) part simulates JPEG2000 and SVC based content adaptation and the entire transmission scenario across different lossy links (e.g., wireless and Internet).

Extraction and Authentication (EA) part consists of preparation for extraction (e.g., up-sizing of resolution adapted content), extraction algorithm (exactly opposite of the WE algorithm), post-processing and authentication using similarity measures such as Hamming distance, normalised cross correlation and etc.

The phase-I of WEBCAM focuses watermarking for scalable coded images. An example of EA results for different quality and joint resolution-quality scalability content adaptations are shown in Figure 2.
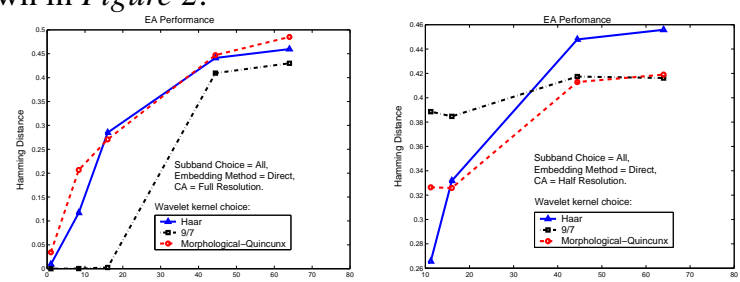

Figure 2: EA Performance examples.

\section{Conclusion}

The proposed WEBCAM provides a good design and evaluation tool for robust watermarking for scalable coded content.

\section{References}

[1] O. Guitart, H. C. Kim, and E. J. Delp. Watermark evaluation testbed. SPIE J. of Elect. Imaging, 15:041106 (13 pages), 2006. 